Received: 27 February 2019

Accepted: 24 June 2019

Published online: 02 August 2019

\title{
X-ray Crystallographic Structure and Oligomerization of Gloeobacter Rhodopsin
}

\author{
Takefumi Morizumi ${ }^{1}$, Wei-Lin Ou ${ }^{1}$, Ned Van Eps ${ }^{1}$, Keiichi Inoue $\mathbb{D}^{3}{ }^{3}$, Hideki Kandori $\mathbb{D}^{4,5}$, \\ Leonid S. Brown $\left(\mathbb{1}^{6}\right.$ \& Oliver P. Ernst $\mathbb{1}^{1,2}$
}

Gloeobacter rhodopsin (GR) is a cyanobacterial proton pump which can be potentially applied to optogenetics. We solved the crystal structure of GR and found that it has overall similarity to the homologous proton pump from Salinibacter ruber, xanthorhodopsin (XR). We identified distinct structural characteristics of GR's hydrogen bonding network in the transmembrane domain as well as the displacement of extracellular sides of the transmembrane helices relative to those of XR. Employing Raman spectroscopy and flash-photolysis, we found that GR in the crystals exists in a state which displays retinal conformation and photochemical cycle similar to the functional form observed in lipids. Based on the crystal structure of GR, we selected a site for spin labeling to determine GR's oligomerization state using double electron-electron resonance (DEER) spectroscopy and demonstrated the $\mathrm{pH}$-dependent pentamer formation of GR. Determination of the structure of $\mathrm{GR}$ as well as its pentamerizing propensity enabled us to reveal the role of structural motifs (extended helices, 3-omega motif and flipped B-C loop) commonly found among light-driven bacterial pumps in oligomer formation. Here we propose a new concept to classify these pumps based on the relationship between their oligomerization propensities and these structural determinants.

Many organisms utilize light-driven transporter proteins to capture light for energy conversion or cell signaling. Rhodopsins are photo-receptive proteins which share a similar overall structure, composed of seven transmembrane $\alpha$-helices (7-TM), and have the chromophore retinal covalently bound to a lysine residue on TM7 via a protonated Schiff base. Light absorption by rhodopsin induces isomerization of a single double bond of the retinal's polyene chain, which triggers structural changes in the protein moiety to perform vital biological functions ${ }^{1}$. Based on primary amino acid sequences, rhodopsins are classified into two groups, type- 1 for microorganisms and type-2 for animals ${ }^{2}$, with predominantly all-trans $\rightarrow 13$-cis and 11-cis $\rightarrow$ all-trans retinal isomerization, respectively.

Microbial rhodopsins are found in Archaea, Bacteria, lower eukaryotes and viruses ${ }^{3}$. They have a variety of functions, such as light-driven ion pumps (bacteriorhodopsin in Archaea; BR), light-gated ion channels (channelrhodopsin in green algae, ChR), and photosensors (sensory rhodopsins I and II in Archaea and Bacteria; SRI and SRII) (Fig. 1) ${ }^{4}$. Although monomeric forms of microbial rhodopsins seem to be the functional unit ${ }^{5}$, several studies have revealed that microbial rhodopsins mostly exist as oligomers in the native membrane environment ${ }^{6,7}$. Previous studies have suggested functional significance of microbial rhodopsin oligomeriza$\operatorname{tion}^{8,9}$, and even functional switch from proton to sodium pumping for KR $2^{10}$; however, the molecular basis that defines the specific oligomeric assemblies for different rhodopsins are unclear and remain to be elucidated $^{11}$. There are a number of biophysical methods to characterize oligomerization ${ }^{12}$. For example, specific oligomeric assemblies of rhodopsins can be crystallized depending on the crystallization conditions, such as dimeric or trimeric structures of $\mathrm{BR}^{13}$, pentameric or hexameric structures of blue-absorbing proteorhodopsin $(\mathrm{BPR})^{9}$, and monomeric or pentameric structures of $\mathrm{KR} 2^{10,14}$. In addition to X-ray crystallography, circular

\footnotetext{
${ }^{1}$ Department of Biochemistry, University of Toronto, Toronto, Ontario, M5S 1A8, Canada. ${ }^{2}$ Department of Molecular Genetics, University of Toronto, Ontario, M5S 1A8, Canada. ${ }^{3}$ The Institute for Solid State Physics, University of Tokyo, Kashiwa, Chiba, 277-8581, Japan. ${ }^{4}$ Department of Life Science and Applied Chemistry, Nagoya Institute of Technology, Showa-ku, Nagoya, 464-8555, Japan. ${ }^{5}$ OptoBioTechnology Research Center, Nagoya Institute of Technology, Showa-ku, Nagoya, 464-8555, Japan. ${ }^{6}$ Department of Physics, University of Guelph, Guelph, Ontario, N1G 2W1, Canada. Takefumi Morizumi and Wei-Lin Ou contributed equally. Correspondence and requests for materials should be addressed to O.P.E. (email: oliver.ernst@utoronto.ca)
} 


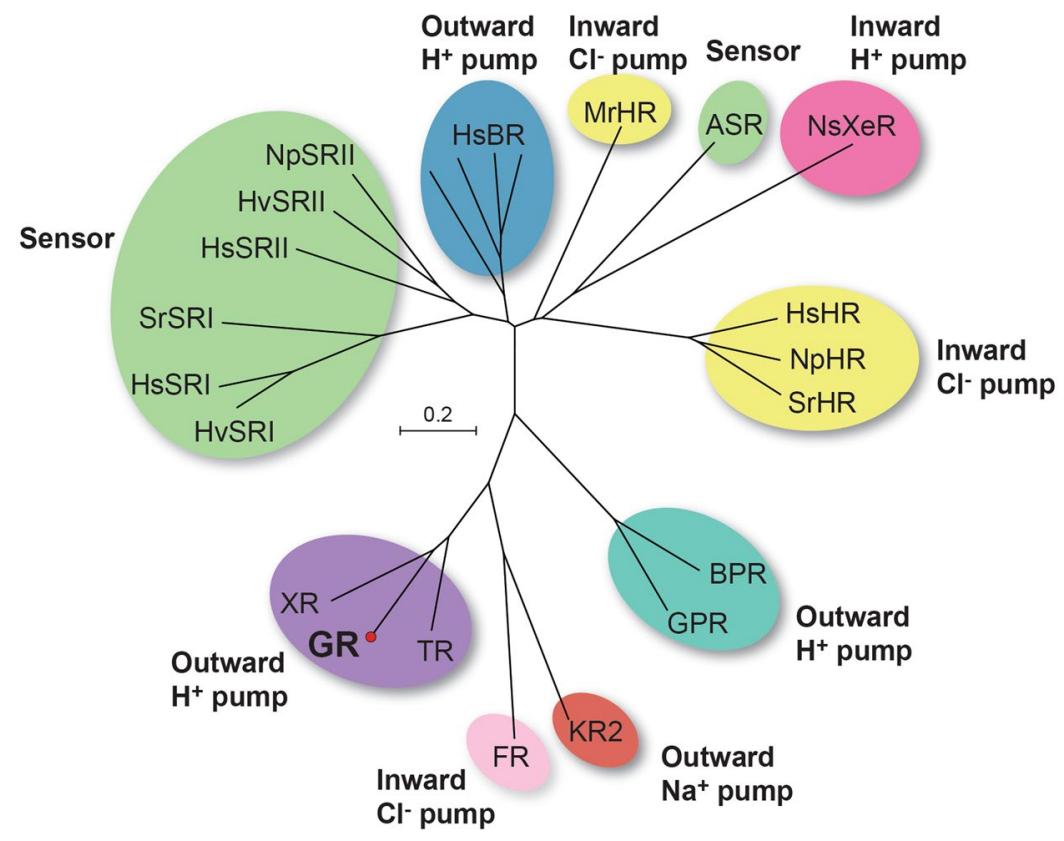

Figure 1. Phylogenetic tree of microbial rhodopsins from Archaea and Eubacteria, representing the phylogenetic relationship between Gloeobacter rhodopsin and related proteins. HsBR, bacteriorhodopsin from H. salinarum; HvSRI, HsSRI, SrSRI, sensory rhodopsin I from Haloarcula vallismortis, H. salinarum, and Salinibacter ruber; HsSRII, HvSRII, NpSRII, sensory rhodopsin II from H. salinarum, Haloarcula vallismortis, Natronomonas pharaonis; MrHR, Mastigocladopsis repens halorhodopsin; ASR, Anabaena sensory rhodopsin; NsXeR, Nanosalinarum sp xenorhodopsin; HsHR, NpHR, SrHR, halorhodopsin from H. salinarum, N. pharaonis, and S. ruber; BPR, blue-absorbing proteorhodopsin; GPR, green-absorbing proteorhodopsin; KR2, sodium-pumping rhodopsin from Krokinobacter eikastus; FR, chloride-pumping rhodopsin from Fulvimarina pelagi; TR, thermophilic rhodopsin from Thermus thermophilus; GR, rhodopsin from Gloeobacter violaceus PCC 7421; XR, xanthorhodopsin from S. ruber. The evolutionary history was inferred using the Neighbor-Joining method ${ }^{64}$. The optimal tree with the sum of branch length $=11.29080174$ is shown. The tree is drawn to scale, with branch lengths in the same units as those of the evolutionary distances used to infer the phylogenetic tree. The evolutionary distances were computed using the Poisson correction method ${ }^{65}$ and are in the units of the number of amino acid substitutions per site. The analysis involved 23 amino acid sequences. All positions containing gaps and missing data were eliminated. There was a total of 189 positions in the final dataset. Evolutionary analyses were conducted with MEGA7 ${ }^{66}$.

dichroism (CD) spectroscopy ${ }^{15,16}$ and high-speed atomic force microscopy (AFM) ${ }^{17,18}$ have been successful in determining the oligomeric states of microbial rhodopsins. Moreover, electron paramagnetic resonance (EPR) spectroscopy combined with site-directed spin-labeling is a powerful tool not only for characterizing conformational changes in microbial rhodopsins, but also for intermolecular distance measurements between protomers providing structural insight into oligomer formation of these 7-TM proteins ${ }^{19,20}$.

Gloeobacter rhodopsin (GR) is a BR-like light-driven proton pump found in unicellular cyanobacterium Gloeobacter violaceus PCC 7421, which lacks thylakoid membranes, and its photosynthetic system co-exists in the cytoplasmic membrane with $\mathrm{GR}^{21}$. Previous studies have suggested it is likely that GR could support ATP synthesis and compensate the poor energy production when the chlorophyll-dependent photosynthesis is low ${ }^{22}$. Interestingly, GR has carotenoid binding capability similar to that found for xanthorhodopsin (XR) ${ }^{23}$. With the ability of binding light-harvesting carotenoid antenna, Gloeobacter cells can utilize various wavelengths of light for energy conversion. In addition, GR shows under specific conditions an inverted proton flux, an interesting feature that other proton pumps commonly applied for optogenetic studies do not have ${ }^{24}$. From recent comprehensive AFM imaging of microbial rhodopsins, the oligomeric state of GR in the reconstituted lipid membrane was proposed to be pentameric ${ }^{17}$, although a previous report concluded that GR has a $\mathrm{pH}$-dependent monomer-trimer transition in detergent micelles ${ }^{25,26}$.

In this study, we present the X-ray crystal structure of GR and discuss the structural features that characterize the XR-like protein subgroup, to which it belongs (Fig. 1). In addition, we identify pentameric oligomerization of GR in detergent micelles using double electron-electron resonance (DEER) spectroscopy ${ }^{27}$, which we demonstrate to be a powerful tool for determining oligomeric assemblies of microbial rhodopsins. From the combination of present experimental results and previous studies, we propose here a new concept to classify microbial rhodopsins by their oligomeric assemblies based on conserved structural motifs. 


\section{Results and Discussion}

Overall structure of GR. Figure 2 a shows the crystal structure of the GR dark state. The structure was determined to $2.0 \AA$ Å resolution (Table 1). Similar to other microbial rhodopsins, the seven transmembrane helices are bundled in a clockwise fashion when observed from the cytoplasmic side, and the all-trans-retinal chromophore is covalently bound to the opsin protein moiety via a Schiff base linkage with the Lys 257 side chain. When crystal structures of Halobacterium salinarum BR (PDB entry 1C3W) and XR (PDB entry 3DDL) were superimposed on GR (Fig. 2b), both root-mean-square deviation (RMSD) values (3.33 with BR and 1.69 with XR) and retinal position indicated that the overall structure of GR is more similar to XR than BR as expected from sequence similarity (Figs 1 and S1).

On the extracellular side, the B-C loop connecting helices B and C has a distinct antiparallel $\beta$-sheet structure similar to most microbial rhodopsins. However, unlike BR which has the $\beta$-sheet oriented towards the middle of its 7-TM helical bundle, the $\beta$-sheet of GR orients oppositely towards helices A and B. As a result, the tip of the $\mathrm{B}-\mathrm{C}$ loop is facing towards the periphery of the GR molecule and the central ion-releasing cavity at the extracellular surface is exposed. This change in orientation of the $\beta$-sheet was first found in the crystal structure of XR and then successively identified in sodium-pumping rhodopsin (KR2) ${ }^{14,28}$, thermophilic rhodopsin (TR $)^{29}$ and eubacterial light-driven chloride-pumping rhodopsin $(\mathrm{ClR})^{29,30}$. In case of KR2 and ClR, an additional N-terminal helix is accommodated close to the extracellular cavity to "cap" the ion-releasing/uptaking region. Another common structural feature of GR, KR2, TR, and ClR are elongated helices A, B and G. As reported for the ClR structure, GR also shares the "3-omega motif" formed by three nonconsecutive aromatic amino acid side chains of Phe38 (helix A), Trp95 (helix B) and Tyr106 (in B-C loop), tethering the B-C loop in the direction of helices A and B (Fig. 2c). Interestingly, these residues are located exactly on the elongated part of helices $A$ and $B$ of these rhodopsins explaining why other BR-type rhodopsins do not share this motif. In contrast to their functional divergence, this structural motif is strictly conserved among different eubacterial pumps, suggesting the motif is not correlated to the function, but to the specific structural arrangement, such as interprotomer interactions. It should be noted that proteorhodopsins only share the elongated helices but lack the 3 -omega motif on it as well as $\beta$-sheet structure in B-C loop. Proteorhodopsins possess a significantly shortened B-C loop which is suggested to have a propensity to form a beta turn in NMR studies ${ }^{31,32}$. Therefore, we hereafter refer to these rhodopsins sharing the features of flipped B-C loop, elongated helices, and 3-omega motif as "XR-type" to be discriminated from both BR-type and proteorhodopsins.

Another structural characteristic distinguishing GR from XR and TR is a relatively tight packing of TM helices at the extracellular side (Fig. 2d). When compared with the XR structure (shown in white), helices A, E, and G are slightly tilted inward towards the center of the helical bundle, resulting in a $\sim 6.6 \AA$ closer positioning of extracellular ends of helices $\mathrm{E}$ and $\mathrm{G}$, whereas no obvious displacements are observed on the cytoplasmic side. At the extracellular side the displacement of helix A also causes a slight displacement of the interacting B-C loop. The repositioning of helix E might be associated with the binding of an additional photoreceptive carotenoid. XR was originally co-crystalized with salinixanthin (SX), the endogenous carotenoid which is considered to be a potential light-harvesting antenna for XR in Salinibacter ruber. GR is also known to bind $\mathrm{SX}^{33}$ as well as the endogenous carotenoid echinenone from $G$. violaceus, both having a 4-keto headgroup ${ }^{34}$, which should locate close to the headgroup of internal all-trans-retinal chromophore for energy transfer. When SX from the XR-SX complex was placed in the analogous position within the GR crystal structure, it fits to the same groove as in XR with a slightly more distant SX headgroup position from retinal chromophore (Fig. 2e). Despite the distant position, however, the SX headgroup in this model showed a subtle steric clash with the inwardly displaced helix E. Given the reported functional binding of SX to GR, helix E might be in the "outward" position as in the XR-SX complex when GR accommodates SX or echinenone. Since GR in our crystals does not have any additional carotenoids, the movement of the extracellular end of helix E is likely to reflect the carotenoid-free structure of GR. It should be noted that other XR-type sodium/chloride pumps, KR2 ${ }^{14}$ and $\mathrm{ClR}^{30}$ have the same helix E position as GR in the absence of carotenoid, and molecular dynamics simulations on TR showed the inward movement of helix $\mathrm{E}$ at higher temperature ${ }^{29}$, suggesting the flexibility of this region.

From the crystal structure of GR, the specific residues responsible for controlling the distance between helices $\mathrm{E}$ and $\mathrm{G}$ could be identified. First, there is a conserved Glu-Arg pair (Glu166-Arg174 in GR, Glu141-Arg152 in $\mathrm{XR}$ ) on the extracellular side of helices $\mathrm{D}$ and $\mathrm{E}$ that can electrostatically interact to modulate the relative position of these helices (Fig. 3a). In addition, another conserved tyrosine residue on helix G of GR (Tyr249) participates in this interaction by forming a hydrogen bonded network with Glu166, whereas the corresponding tyrosine residues of XR and TR do not (Fig. 3b). Furthermore, we found a steric constraint presented by a Phe residue in XR and TR, at the position homologous to Val224 on helix F of GR, that could interfere with the movement of helices F and G (Fig. 3c). Located between helices F and G, the bulky side chain of phenylalanine provides (in case of XR and TR) a steric constraint on helix G so that it cannot tilt inwardly as it does in GR. As a result of these interactions, helices $\mathrm{E}$ and $\mathrm{G}$ come closer in GR but not in XR and TR.

Structure of retinal binding pocket and the proton transport pathway of GR. When it comes to the proton transport pathway, including the retinal binding pocket, GR has an organization more similar to XR than BR. The three possible regions of hydrogen-bonded network involving the side chains of polar residues and the observed water molecules in the pathway are shown in Fig. 4. It should be noted that the number of water molecules observed to participate in the network depends on the resolution of crystal structures as well as the hydration itself. Therefore, we used water molecules of BR structures that had been confirmed to exist in the internal pathway (wat-401 to 406, 501 and 502) to compare with waters clearly observed in the GR structure (Fig. S2).

First, in the cytoplasmic region of GR, the functionally important water molecules (wat-501, 502) are conserved in both XR and BR. In BR, a proton donor Asp96 is connected to wat-502 through a hydrogen-bonded network via Thr46, whereas the corresponding putative proton donor in GR and XR is replaced by Glu (Glu132 
(a)
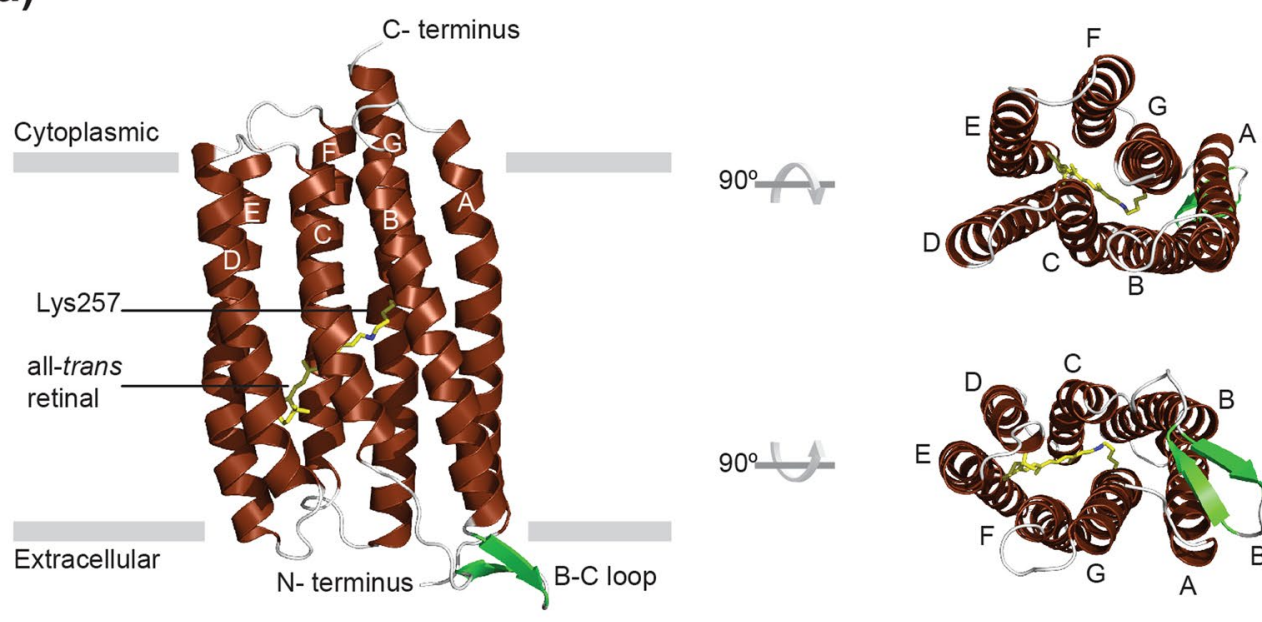

(b)

GR vS BR $\operatorname{RMSD}=3.33 \AA$
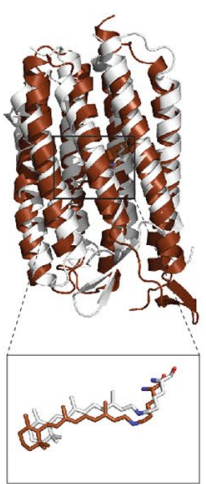

GR vs XR $\mathrm{RMSD}=1.69 \AA$

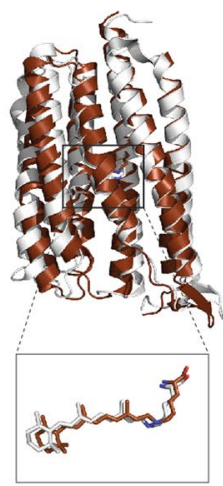

(c)
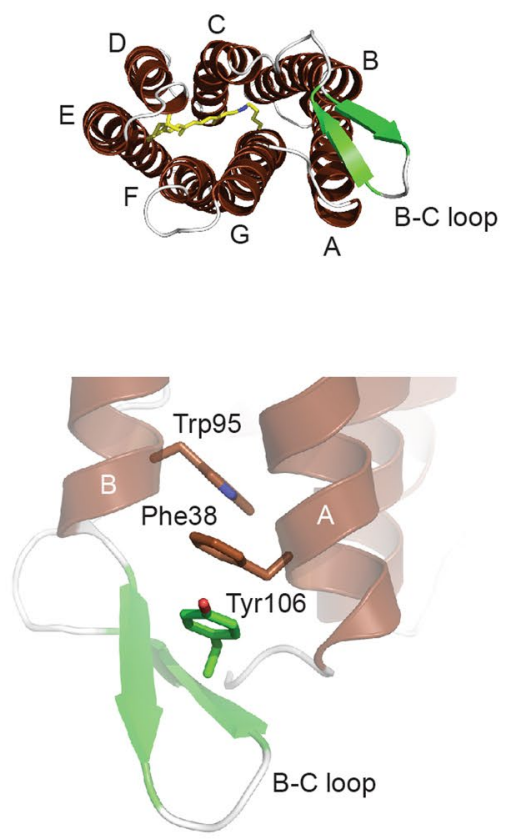

(e)

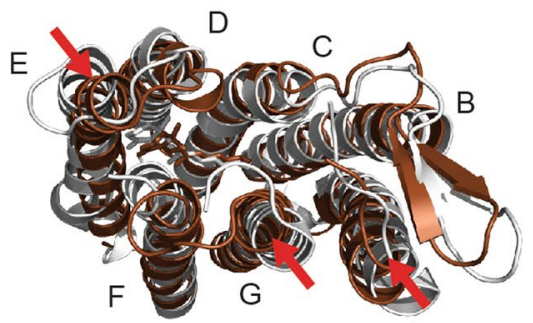

A

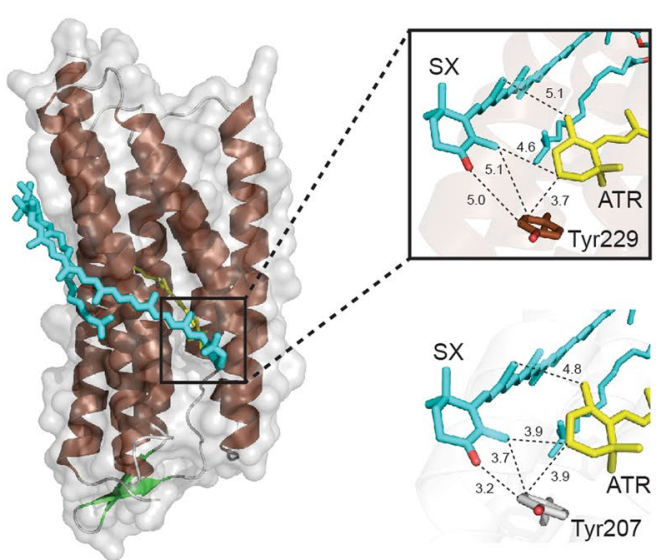

Figure 2. Structural details of GR. (a) Overall structure of GR shown in cartoon representation viewed parallel to the membrane (left), from intracellular side (right, top) and from extracellular side (right, bottom). GR consists of seven transmembrane helices (TM A to TM G, shown in brown) connected by interhelical loops (shown in white) on both sides of the membrane. The $\beta$-strands in the B-C loop are shown in green. Alltrans-retinal, depicted by stick models, is covalently linked to Lys 257 via a protonated Schiff base (shown in yellow). (b) Superimposed structures of GR (brown) and H. salinarum BR (white, PDB entry 1C3W), and GR and XR (white, PDB entry 3DDL), respectively. (c) 3-omega motif of GR formed by $\pi$-stacking interactions of the side chains of aromatic residues in TM A (F38), TM B (W95) and B-C loop (Y106). (d) Extracellular side of superimposed GR (brown) and XR (white) structures with the XR-bound SX molecule (cyan). The helix displacements are indicated by red arrows. (e) Potential carotenoid binding site in GR. Salinixanthin (SX) from the crystal structure of XR is superimposed onto the structure of GR. The magnified views compare the location of SX and the retinal in GR (top) and XR (bottom). 


\begin{tabular}{|c|c|}
\hline Resolution $(\AA)$ & $42.79-2.0$ \\
\hline Space Group & C 2221 \\
\hline \multicolumn{2}{|l|}{ Unit Cell Dimensions } \\
\hline $\mathrm{a}(\AA)$ & 54.25 \\
\hline $\mathrm{b}(\AA)$ & 129.25 \\
\hline$c(\AA)$ & 82.65 \\
\hline Alpha (degrees) & 90 \\
\hline Beta (degrees) & 90 \\
\hline Gamma (degrees) & 90 \\
\hline Molecules in the asymmetric unit & 1 \\
\hline Solvent content (\%) & 50 \\
\hline Unique reflections & 19313 \\
\hline Completeness for range (\%) & 96.37 \\
\hline Rcryst (\%) & 23.03 \\
\hline Rfree $(\%)$ & 24.44 \\
\hline Rmerge (\%) & $4.4(21.6)$ \\
\hline R.m.s deviations (bond lengths) & 0.009 \\
\hline R.m.s deviations (bond angles) & 0.85 \\
\hline Ramachandran outliers (\%) & 0.82 \\
\hline Clashscore & 3.29 \\
\hline Average B-factor & 38.72 \\
\hline \multicolumn{2}{|c|}{ Data collected at the Advanced Photon Source (APS), Argonne National Laboratory } \\
\hline X-ray source & 23ID-D \\
\hline Detector type & Pilatus 6M \\
\hline Collection software & In house software package (JBluIce) \\
\hline Data indexed, collected, scaled & In house software package, XDS, Aimless \\
\hline Search model PDB ID & 3DDL \\
\hline Model alignment used & Blast web server \\
\hline Molecular replacement program & Balbes \\
\hline Refinement software & Phenix.Refine (in the Phenix suite) \\
\hline Model building software & COOT \\
\hline
\end{tabular}

Table 1. Crystal data, data collection, and refinement statistics.

(a)

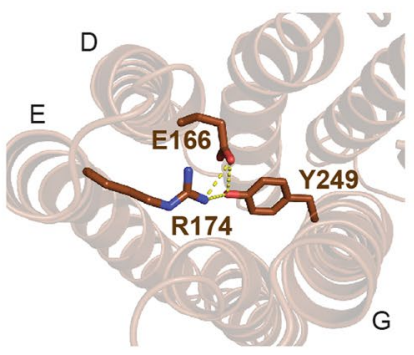

(b)

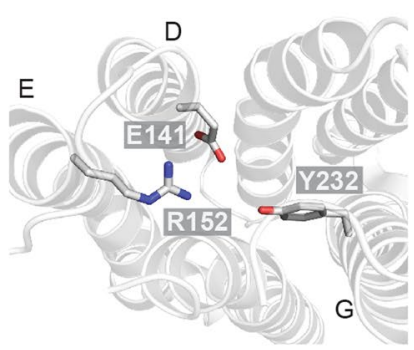

(c)

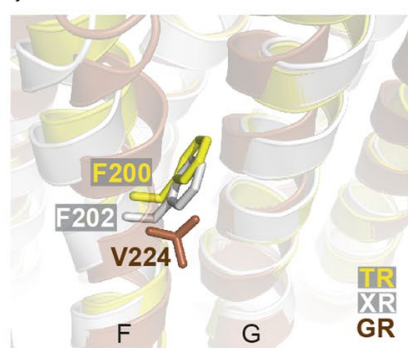

Figure 3. Conserved Glu-Arg salt bridge on the extracellular side of helices D and E; E166-R174 in GR (a) and E141-R152 in XR (b). (c) Steric interference of movement of helices F and G by Phe residues on helix F in XR and TR.

and Glu107, respectively) and interacts directly with wat-502 (Fig. 4a). This structure explains the previous observation of the long-range structural perturbation of Glu132 upon retinal photoisomerization at $77 \mathrm{~K}^{35}$. In contrast, the location and hydrogen-bonded network involving wat-501 in GR and XR are almost the same as in BR.

Next, on the extracellular side of the Schiff base of GR, only one water molecule (wat-402 in XR) is mediating the hydrogen-bonded network among Asp121, Asp253 and retinal Schiff base, whereas BR has a water triad (wat-401, 402 and 406) among the corresponding Asp85, Asp212 and the Schiff base (Fig. 4b). Since the water triad in BR has been repeatedly found in structures with comparable resolution $(2.0 \AA$ to $2.3 \AA$, PDB entries 5BR5 and 1IW6), it is likely that GR and XR accommodate only one water molecule instead of the triad. In fact, the side chain of Asp253 in GR comes closer to other residues narrowing the cavity to establish this simplified network with a single water molecule. This water molecule probably forms a strong hydrogen bond with the 
(a)
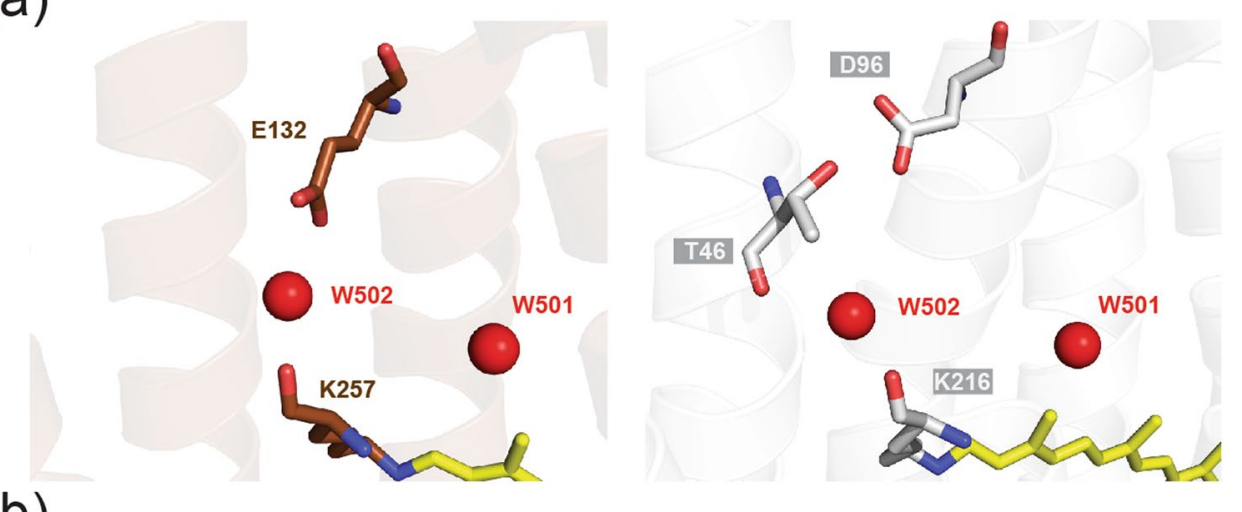

(b)
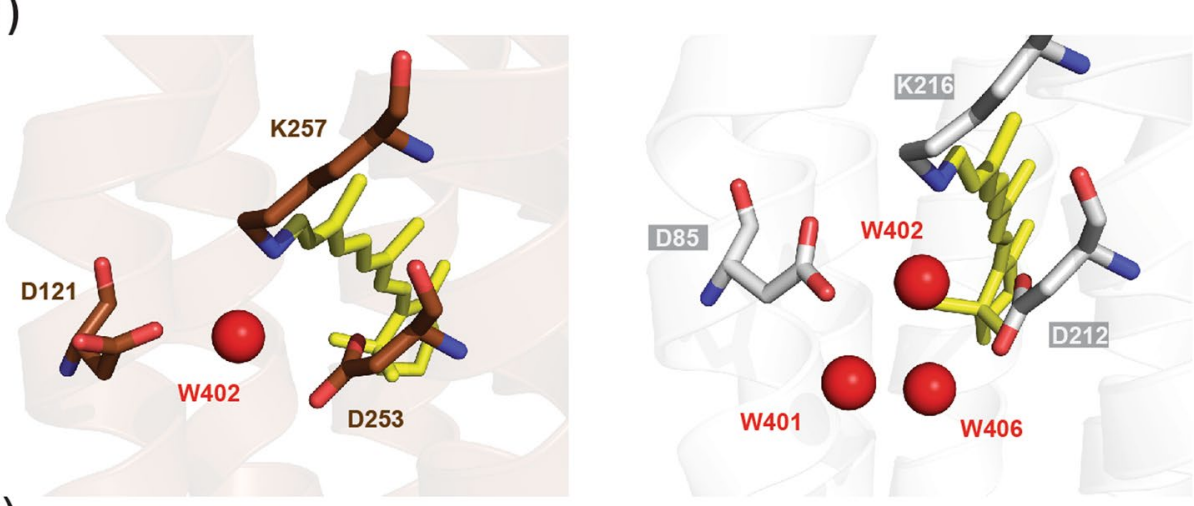

(C)
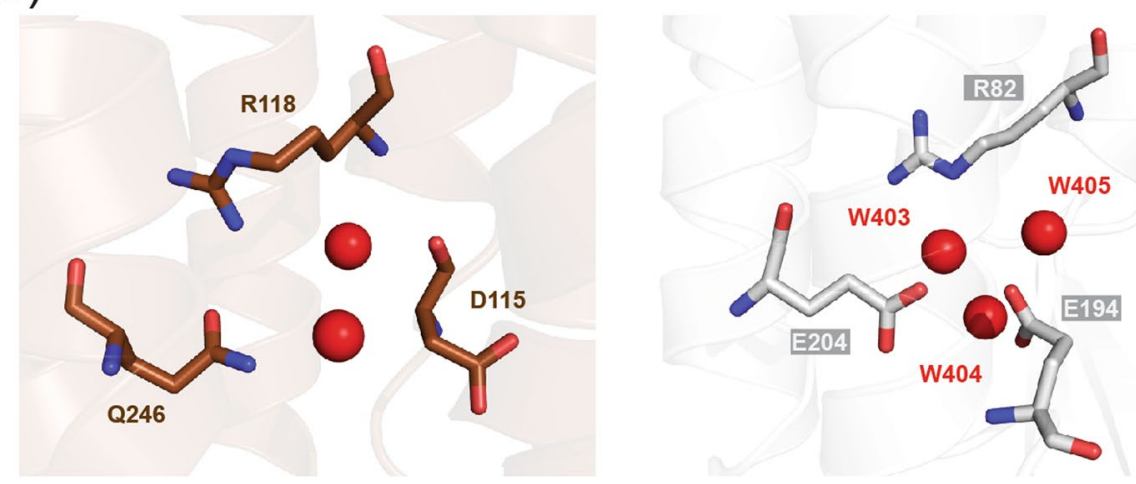

Figure 4. Comparison of three internal cavities in proton transporting pathway of GR (brown) and BR (white). (a) Cytoplasmic side of the Schiff base region. (b) Extracellular side of the Schiff base region. (c) Cavities near the extracellular surface.

counterion of the Schiff base as reported by low-temperature FTIR spectroscopy ${ }^{35}$. In addition, similar to XR, GR also has the Asp121-His87 pair as the counterion (Asp85 in BR) of the Schiff base, confirming it is a conserved feature in XR-type proton pumps (XR/GR/TR) as well as proteorhodopsins (BPR/green-absorbing proteorhodopsin, GPR/Exiguobacterium sibiricum rhodopsin, ESR). The linkage between the titratable Asp-His pair and the Schiff base would explain the $\mathrm{pH}$ dependent shift of the absorption maximum in GR and the GPR dark state spectrum $^{25,36}$.

Lastly, in the extracellular region where the proton release during the photocycle occurs, only two water molecules are found in GR compared with three water molecules in BR (called wat-403, 404 and 405). Also, the surrounding polar residues for this cavity (Asp115, Arg118 and Gln246 in GR) are connected more directly than in BR, accommodating fewer water molecules (Fig. 4c). The most prominent difference found in GR (also in XR/ TR) is the lack of the Glu194/Glu204 pair of BR. The hydrogen-bonded network including the Glu194/Glu204 pair and water molecules wat- 403 to 405 has been considered to be responsible for the proton release from the extracellular side in the BR photocycle in combination with the rearrangement of the Arg82 side chain ${ }^{37,38}$. XR-type proton pumps lack this Glu/Glu pair, and the glutamate at position 204 is replaced by glutamine (Gln246 in GR). By all these changes, GR establishes a new hydrogen-bonded network connecting Arg118, Gln246, and Asp115 (Fig. 4c). Since Gln246 cannot serve as the proton donor, it is reasonable to assume that Asp115 could take over the proton release function at the extracellular surface instead of the Glu194/Glu204 pair employed in 
BR. However, confirmation with mutagenesis would be required. The homologous aspartic acid residue is also conserved in XR and TR but not in proteorhodopsins, although they lack the Glu/Glu pair. Instead, another glutamate on helix D, Glu124 in BPR participates in the hydrogen-bonded network, locating the position near to the Glu194 of BR. As mentioned in the previous section, this glutamate in XR-type proton pumps is tightly associated with arginine on helix E (Glu166-Arg174 salt bridge in GR, Fig. 3a) and therefore would not participate in the network. Interestingly, ESR lacks the homolog of Glu194, but retains Glu204 of BR as Glu214.

Raman spectroscopy and time-resolved laser spectroscopy. Raman spectroscopy and time-resolved laser spectroscopy in the visible range were performed to probe the GR dark state and the photocycle in the crystals (Fig. S3). The plate-like crystals were grown in bicelles at pH 3.4 in the dark (see below) and then harvested for spectroscopic studies. The Raman bands of $\mathrm{C}=\mathrm{C}$ and $\mathrm{C}-\mathrm{C}$ stretching vibrations (compare the position of the peak at $1529 \mathrm{~cm}^{-1}$ for the former and the ratio of the peaks at 1200 and $1170 \mathrm{~cm}^{-1}$ for the latter) in crystals look very similar to those in proteoliposomes at $\mathrm{pH} 9$ but not at $\mathrm{pH} 3$. This suggests that a form of GR with retinal configuration and environment similar to those of the alkaline proton-pumping state in lipids is expected to be found in crystals (Fig. S3a). This assumption is supported by the photocycle kinetics of crystalline GR probed by time-resolved laser spectroscopy in the visible range (flash-photolysis). The photocycle of the alkaline proton-pumping form of GR in lipids is dominated by the late red-shifted O intermediate detectable at $620 \mathrm{~nm}$ (Fig. S3b), with strongly pH-dependent kinetics ${ }^{39}$. As reported previously, such accumulation of the late red-shifted $\mathrm{O}$ intermediate is diminished when the Schiff base carboxylic counterion is neutralized either by mutation or by protonation at low $\mathrm{pH}$ not only in GR, but also in homologous proton pumps XR and $\mathrm{PR}^{40,41}$, which correlates with the disappearance of proton transport and the respective $M$ intermediates. Figure S3b shows laser light-induced difference light absorption kinetics of crystalline GR measured at $620 \mathrm{~nm}$ compared with those of liposome-reconstituted GR measured earlier ${ }^{39,42}$. The strong accumulation of the red-shifted intermediate and the kinetics of its rise and decay suggests that the photocycle of crystalline GR is similar to that of the alkaline form of GR in lipids at near neutral $\mathrm{pH}$, further confirming similarity of crystalline GR to the functional state of GR in membranes.

pH-dependent color transition and oligomer formation. One of the primary methods to characterize microbial rhodopsins is to observe their optical absorption spectrum. In case of BR, solubilization by Triton X-100 produces monomeric BR in solution with a blue-shift of the absorption maximum from 568 to $551 \mathrm{~nm}^{43,44}$. In contrast, GPR is known to show a red-shift from 521 to $535 \mathrm{~nm}$ when GPR monomers are formed by solubilization with Triton X-100 or $\mathrm{OG}^{36}$, but GPR can retain its higher oligomeric state in milder detergent, such as $\mathrm{DDM}$ at $\mathrm{pH} 8.2^{19,45}$. In addition, GPR shows a $\mathrm{pH}$ dependent red-shift from 520 to $540 \mathrm{~nm}$ at acidic $\mathrm{pH}^{46}$. These results suggest the relationship between oligomeric state and absorption spectral change, probably based on the $\mathrm{pKa}$ shift of the retinal counterion. Similar to GPR, GR shows a pH-dependent red-shift of the absorption maximum from alkaline $\mathrm{pH}\left(8.0, \lambda_{\max }=545 \mathrm{~nm}\right)$ to acidic $\mathrm{pH}\left(3.0, \lambda_{\max }=560 \mathrm{~nm}\right)$ in DDM (Fig. 5a). Moreover, we could demonstrate the molecular size change by size-exclusion chromatography (SEC) at different pH (Fig. 5b), consistent with a previous study of GR that showed a pH-dependent oligomer-monomer transition in DDM and $\mathrm{GR}$ monomers at acidic $\mathrm{pH}^{25}$. At acidic/alkaline $\mathrm{pH}$, the calculated molar masses from the $\mathrm{SEC}$ retention volume of the peaks were $87 \mathrm{kDa} / 239 \mathrm{kDa}$, respectively. Given that the empty DDM micelle size is $\sim 50 \mathrm{kDa}$, it is likely that $\mathrm{GR}(33 \mathrm{kDa})$ is monomeric at $\mathrm{pH} 3.0$ and becomes oligomeric at $\mathrm{pH}$ 8.0. We then performed negative stain electron microscopy (EM) imaging to visualize the oligomer-monomer transition of GR (Fig. 5c,d). The samples collected from SEC peaks at $\mathrm{pH} 8.0$ and 3.0 were directly applied to the carbon-coated EM grids and negatively stained by $2 \%$ uranyl-acetate. The images clearly showed homogeneous oligomers at $\mathrm{pH} 8.0$, whereas most of them are dissociated and became roughly one-third in particle size at $\mathrm{pH} 3.0$, consistent with the change of the SEC elution profile due to changes in mass.

Probing oligomeric states of GR by EPR spectroscopy. Recent comprehensive AFM imaging of microbial rhodopsins showed that GR is pentameric in the reconstituted lipid membrane ${ }^{17}$, although a previous report concluded from SEC that GR has pH-dependent monomer-trimer transition in DDM micelles ${ }^{25}$. To investigate the molecular basis of oligomerization in GR, it is important to clarify if GR can have both trimeric and pentameric states. Therefore, we applied EPR spectroscopy to probe the $\mathrm{pH}$-dependent change of the GR oligomerization state in micelles.

To determine the best spin labeling site for intermolecular distance measurement, the crystal structure of GR has been superimposed with the crystal structure of trimeric BR (Fig. 6b), pentameric KR2 (Fig. 6c) and hexameric BPR (Fig. 6d). Gly67 on the cytoplasmic A-B loop has been selected for spin-labeling for EPR/DEER experiments. The spin labeling on site 67 will theoretically yield no distance for a GR monomer, a single $\sim 37 \AA$ distance for a trimer as in BR, two distinguishable distances of $\sim 18 \AA$ and $\sim 29 \AA$ for a pentamer as in KR2, and three $\sim 16 \AA, \sim 28 \AA$ and $\sim 31 \AA$ for a hexamer as in BPR. A single cysteine mutant (G67C) of GR was expressed, purified and collected from SEC peaks at both $\mathrm{pH} 8.0$ and $\mathrm{pH} 3.0$, and then spin labeled to generate a R1 nitroxide side chain. From the continuous wave (CW) EPR line-shape analysis, the samples at both pH 8.0 and pH 3.0 were confirmed to be appropriately spin-labelled. Only at pH 8.0 the CW EPR spectrum is strongly broadened due to the dipolar interaction between nitroxide spin-labels, suggesting a close proximity of the spin labels caused by oligomerization of GR (Fig. 6e). It is suggested by SEC that the majority of GR is monomeric under acidic conditions, and this is also observed in the CW-EPR experiment as the dipolar broadening effects diminished at $\mathrm{pH}$ 3.0. The distance between spin-labels was measured by DEER spectroscopy and the oligomeric state of GR was determined accordingly. Figure $6 \mathrm{f}$ inset shows the background corrected DEER dipolar evolution functions reflecting the proximity between spin-labels. The sample showed clear DEER signals at $\mathrm{pH}$ 8.0, but no signal at $\mathrm{pH} 3.0$, indicating that GR forms oligomer at $\mathrm{pH} 8.0$ and is monomeric at $\mathrm{pH} 3.0$ resulting in no interaction 
(a)

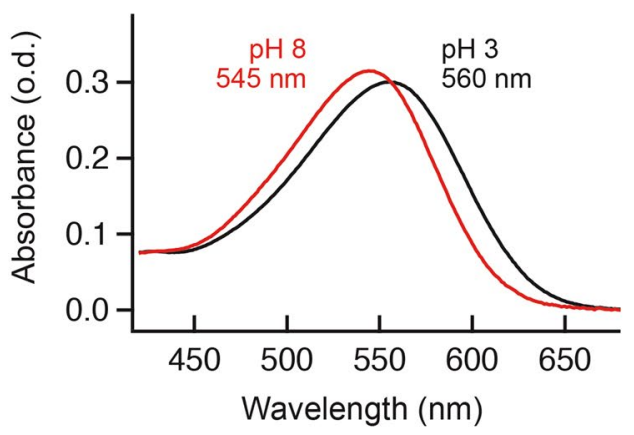

(c)

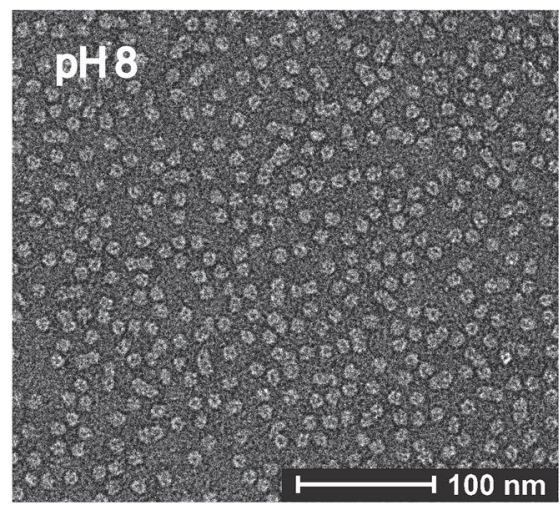

(b)

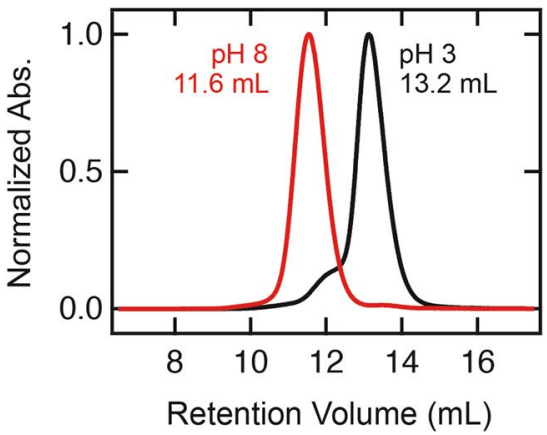

(d)

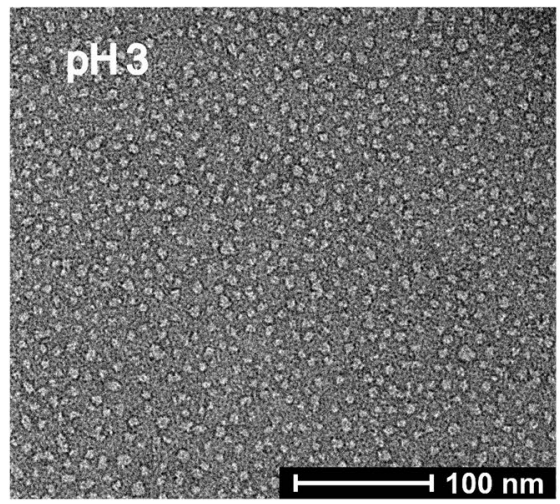

Figure 5. (a) UV-Vis spectra of GR at different $\mathrm{pH}$ conditions. Absorption maxima are indictaed. (b) Size exclusion chromatogram of GR at different $\mathrm{pH}$ conditions. Peak elution volumes are indictaed. (c,d) EM images of GR at pH 8.0 and 3.0, negatively stained with $2 \%$ uranyl acetate (Scale bar, $100 \mathrm{~nm}$ ).

between spins. The DEER signal at pH 8.0 could be further analyzed and two distinct distance peaks at $19 \AA$ and $29 \AA$ were obtained from the distance distribution shown in Fig. $6 f$. These two distances are in close agreement with the two expected distances from the pentameric model of GR, as shown in the cartoon (Fig. 6c). In an analogous approach, two DEER distance peaks with a similar distance ratio characteristic for pentamers were reported previously for the CorA pentamer ${ }^{47}$. Therefore, we can conclude that the oligomeric state of GR formed at $\mathrm{pH} 8.0$ is pentameric, whereas GR is monomeric at pH 3.0 as shown by the lack of intermolecular distances.

Oligomerization of microbial rhodopsins. Oligomerization has specific impact on the function of membrane proteins. Microbial rhodopsins are often found as oligomers in native and artificial membranes. Even though many microbial rhodopsins are functional as a monomer, it is unclear why homo-oligomerization would be beneficial for the organism. It has been observed that the trimeric assemblies of BR show greater thermal stability than monomeric $\mathrm{BR}^{48}$. Interestingly, the crystal structures of different species, i.e., of blue light-absorbing proteorhodopsin (BPR) show pentameric (HOT75BPR) and hexameric (Med12BPR) arrangements ${ }^{9}$, which is very different from the arrangement of $\mathrm{BR}$ trimers. These pentameric/hexameric arrangements of proteorhodopsins are also shown by AFM and NMR studies ${ }^{17,49}$. Specifically, the main cross-protomer interactions occur between residues of helices $\mathrm{A}, \mathrm{B}$ and $\mathrm{C}$ for both pentamers and hexamers in BPR, while the interactions are mainly contributed by helix B and D in BR trimer (Fig. S5). When the interacting helices between protomers of BPR pentamer and hexamer are superimposed, it is obvious that the overall structure of these helices in each protomer is almost identical (Fig. S5d, right half) and only a change of interaction angle by tilting is observed (Fig. S5d, left half). These facts suggest that a pentamer could transform into a hexamer by a small change of interaction, whereas transforming a trimer would require much larger change of protomer organization. Also, the similarity of cross-protomer arrangement between pentamers of sodium pump (KR2) $)^{10,14}$ and proton pump $(\mathrm{BPR})^{9}$ suggests that regardless of the function of the pumps, the interacting helices are conserved among the pentameric microbial rhodopsins.

Our DEER-EPR analysis reveals that GR forms pentamers in micelles, which is consistent with a previous AFM and CD analysis in lipid environment ${ }^{17}$. Due to genetic proximity, the GR pentamer model was acquired by superimposing the GR structure onto the KR2 pentamer to find the potential cross-protomer interacting residues between helices A and B (Fig. S6). A histidine residue on helix B (His87) is facing towards the backbone of Ser46 on helix A of the neighboring protomer. As described above, this histidine is well-conserved in both XR-type proton pumps (XR/GR/TR) and proteorhodopsins (BPR/GPR/ESR), paired with aspartate in the same protomer and working as the counterion of the Schiff base. A previous mutation study on GR showed that His87 is the residue titrated by $\mathrm{pH}$ change and that breaking the His87-Asp121 interaction at low $\mathrm{pH}$ translates into changes 
(a)

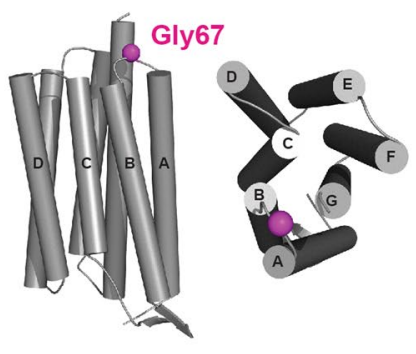

(c)

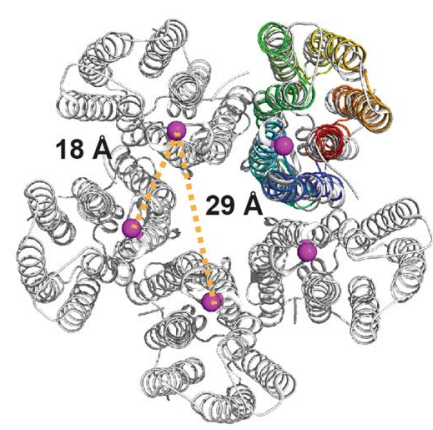

(e)

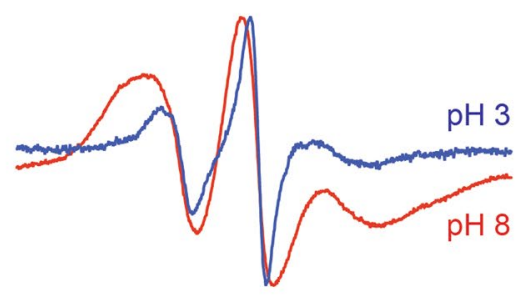

(b)

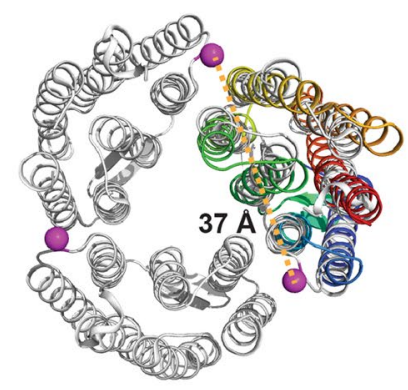

(d)

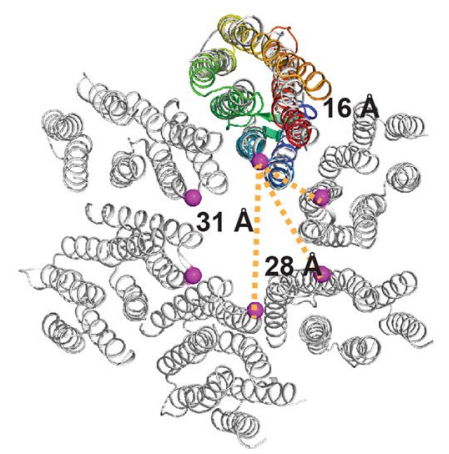

(f)

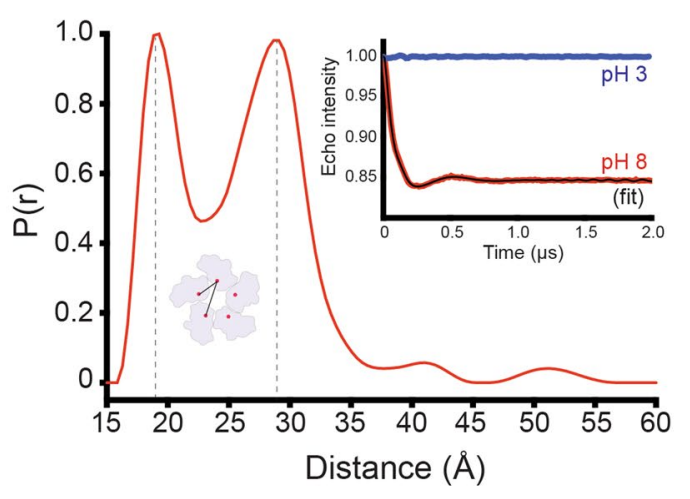

Figure 6. EPR analysis of the oligomeric states of spin labeled GR-67R1. (a) Spin-labeling site on GR viewed parallel to the membrane (left) and from the cytoplasmic side (right). The $\alpha$-carbon of Gly67 is colored magenta and shown as a sphere. (b-d) Superimposed structure of GR and HsBR (b) GR and KR2 (c) and BPR (d). $\alpha$-carbon of the residues equivalent to Gly67 in GR are shown as a sphere. (e) Room temperature CW EPR spectra of GR-67R1 in $0.05 \% \mathrm{DDM}$ at $\mathrm{pH} 3.0$ and $\mathrm{pH}$ 8.0. Dipolar broadening in the $\mathrm{pH} 8.0$ spectrum is clearly observed. (f) DEER distance distribution of GR-67R1 at pH 8.0. Inset: baseline-corrected DEER traces.

of the quaternary structure yielding monomers as the dominant form ${ }^{25}$. Therefore, it is reasonable that His 87 plays an important role in the protomer interacting surface. Interestingly, another cross-protomer polar residue pair in this interface near the extracellular surface (Asn43-Asn114 in GR) is conserved in all XR-type rhodopsins (XR/GR/TR and KR2/ClR), but not in proteorhodopsins (BPR/GPR/ESR). The prominent structural differences between these two groups are that the XR-type rhodopsins have a B-C loop with distinct antiparallel $\beta$-sheet structure and also share the 3-omega motif, which tethers the B-C loop in the direction of TM helices A and B. The KR2 pentamer found in the crystal and the GR pentamer model strongly suggest the presence of additional interaction between the "flipped" B-C loops of each protomer (Fig. S6) to fix the pentameric organization.

In Fig. 7, a new concept to regroup bacterial pumps based on the relationships between oligomeric states and the conserved structural determinants, i.e. extended helices, 3-omega motif and flipped B-C loop, is summarized. As shown in Fig. $7 \mathrm{a}$, archaeal rhodopsins $\left(\mathrm{AR}^{50}, \mathrm{BR}^{18,51}, \mathrm{SR}^{17}\right.$, and $\left.\mathrm{HR}^{17,52}\right)$ form trimeric oligomers exclusively, whereas eubacterial rhodopsins form both trimeric (Anabaena sensory rhodopsin (ASR) ${ }^{17,20,53}$, xenorhodop$\sin (\mathrm{XeR})^{17,54}$ and M. repens halorhodopsin (MrHR; Besaw, Ou, Morizumi, Vasquez, Miller, Ernst, unpublished observation) and pentameric/hexameric (GR, TR, KR2, FR, BPR, and GPR) oligomers. Therefore, the propensity to form pentamers/hexamers happens to be acquired at the point where Bacteria and Archaea branched. It is worth noting that ASR, XeR and MrHR have close sequence similarity to archaeal sub-family. 
(a)

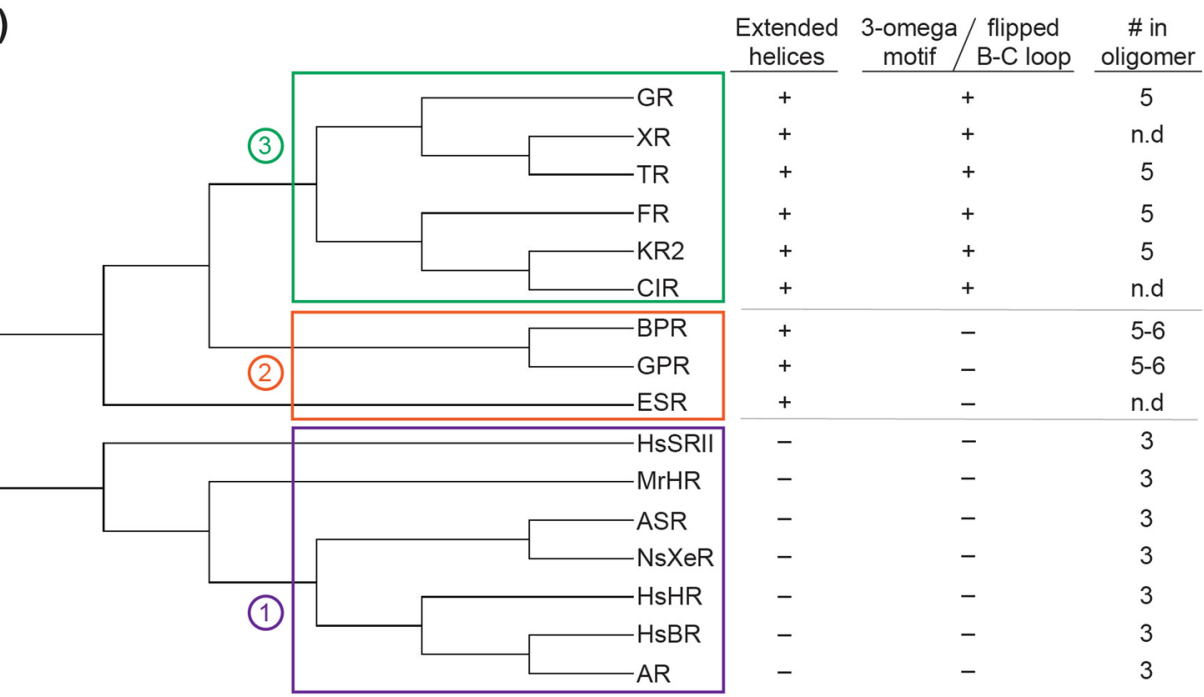

(b)

(1)

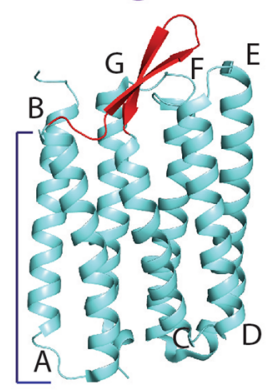

$\downarrow$

Trimer

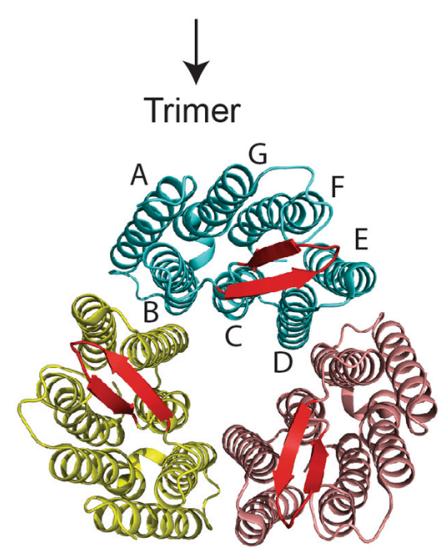

(2)

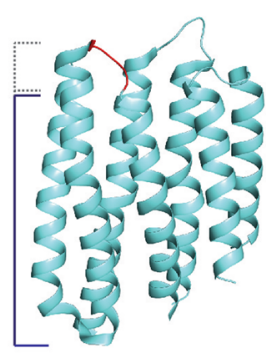

(3)

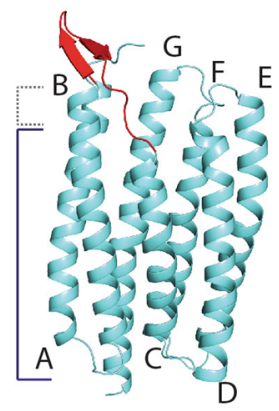

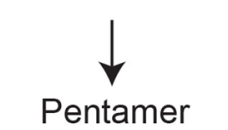

Hexamer

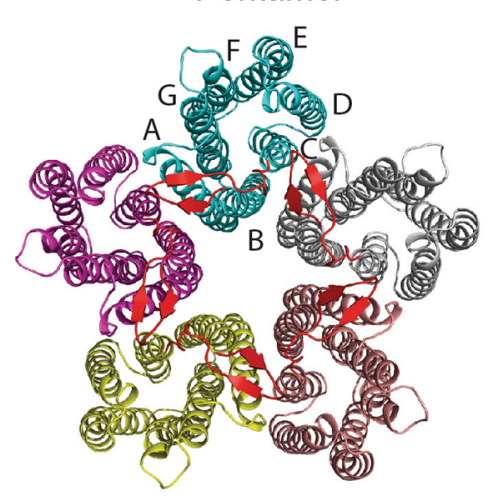

Figure 7. Summary of relationships between the evolutionally altered structural features and oligomerization propensities. (a) Phylogenetic tree and structural motifs. The important branches are numbered: extension of the interface helices as 1, acquisition of 3-omega motif and flipped B-C loop as 2 and the functional divergence in XR-type proteins as 3. (b) (upper) Crystal structures of BR (left, PDB), BPR-Med12 (middle) and GR (right) viewed parallel to the membrane. (lower) Trimeric organization of BR (left) and modelled pentameric organization of GR (right). B-C loops are colored in Red.

Using available structural data on microbial rhodopsins, we elaborate on the structural determinants causing differences in their oligomerization propensities. First, both archaeal and trimeric eubacterial rhodopsins have relatively short TM helices A, B and G (Fig. 7b, left) compared to extended helices of other non-trimeric eubacterial rhodopsins/proteorhodopsins (Fig. 7b, middle), and XR-type rhodopsins (Fig. 7b, right). Judging from the phylogenetic tree of microbial rhodopsins (Fig. 7a), it is clear that the extension of these helices was acquired at the point where non-trimeric eubacterial rhodopsins (group 2 and 3) branched from trimeric rhodopsins (group 1). Therefore, it is likely that this was a key-event that occurred which converted trimeric to pentameric/ 
hexameric receptors. Second, XR-type rhodopsins have a B-C loop tethered outwards by the 3-omega motif (Fig. 2c) which is formed by an extension of TM helices A and B (group 3: Fig. 7b, right). In contrast, archaeal rhodopsins and trimeric eubacterial rhodopsins have the B-C loop oriented towards the center of the TM helical bundle (group 1: Fig. 7b, left). Interestingly, proteorhodopsins lack the $\beta$-sheet structure of the B-C loop as well as the 3-omega motif (group 2: Fig. 7b, middle). Therefore, proteorhodopsins as group 2 (pentameric/hexameric) and XR-type rhodopsins as group 3 (pentameric) receptors were branched by the acquisition of the outwards tethered B-C loop. The KR2 pentamer and our GR pentamer model show that the protomer interface for oligomerization is composed of helices A, B and C, which includes the interaction between B-C loops (Fig. S6). The lack of specific structural motifs at the proteorhodopsin protomer interface might account for their propensity to form hexamers ${ }^{6,17}$ as well as the hexameric crystal structure of BPR. In fact, the hexameric structure of BPR-Med12 (PDB:4JQ6) does not have any interactions within this region, however, the pentameric structure of BPR-HOT75 (PDB:4KLY) has interactions between its long N-terminus and the B-C loop of the neighboring protomer (Fig. S5d).

The reported crystal structure of GR gives insights into structural changes that may occur upon pentamer formation and/or the binding of carotenoid molecules. The high-resolution structure enabled us to confirm the altered hydrogen bonding network in XR-type proton pumps as a common feature, and highlighted candidate residues important for $\mathrm{pH}$ mediated oligomerization and proton releasing function. Because both EPR distance measurements and previous AFM results show a pentameric GR state, we find that GR oligomeric states are insensitive to surrounding detergent or lipid environments.

Although further comprehensive characterization would be needed to define the molecular mechanism of oligomerization, there are no known exceptions for the correlation between the direction of B-C loop and the proposed trimer-pentamer oligomerization. This may be essential knowledge for protein engineering of microbial rhodopsins to control oligomeric states which potentially modulate optogenetic functions of the receptors.

\section{Materials and Methods}

Expression and purification of GR. Wild-type and mutant GR proteins with a $6 x$ histidine tag at the C-terminus were expressed in E. coli strain C43(DE3) as described previously ${ }^{39,55}$. The cells were induced with $1 \mathrm{mM}$ IPTG for $6 \mathrm{~h}$ at $37^{\circ} \mathrm{C}$ and all-trans-retinal was added to final concentration of $5 \mu \mathrm{M}$. The GR proteins were solubilized with $1.0 \% \mathrm{n}$-Octyl- $\beta$-D-glucopyranoside (OG) or $\mathrm{n}$-dodecyl- $\beta$-D-maltoside (DDM) at room temperature followed by ultracentrifugation at $100,000 \times \mathrm{g}$ to remove unsolubilized material. For GR purification, the supernatant was loaded onto a TALON $\mathrm{Co}^{2+}$ column in the solubilizing buffer and eluted by lowering $\mathrm{pH}$. GR fractions were further purified on a Superdex 200 10/300 GL column with running buffer (20 mM HEPES, $\mathrm{pH} 7.5,300 \mathrm{mM} \mathrm{NaCl}$ containing $1.0 \%$ OG or $0.04 \% \mathrm{DDM})$. The peak fraction of GR was collected and used immediately without freezing. The UV-vis absorption spectrum was measured using a CARY $60 \mathrm{UV}$-Vis spectrophotometer equipped with a Peltier temperature control unit (Agilent Technologies) at $15^{\circ} \mathrm{C}$.

Crystallization of GR. GR crystals were grown in bicelles using the hanging drop method similar to what was previously described for bacteriorhodopsin crystallization ${ }^{56,57}$. The purified GR in $1 \%$ OG was concentrated to $\sim 15 \mathrm{mg} / \mathrm{mL}$ and mixed with $24 \%$ (w/v) DMPC:CHAPSO bicelles in a 1:2 ratio (final $8 \%$ bicelles) on ice where the mixture remains liquid and kept for $30 \mathrm{~min}$ to equilibrate. Crystallization was initiated by mixing $4 \mu \mathrm{L}$ of the protein/bicelle mixture with $1.4 \mu \mathrm{L}$ of well solution (2.6-2.8 $\mathrm{M} \mathrm{NaH}_{2} \mathrm{PO}_{4} \mathrm{pH} 3.4,180 \mathrm{mM}$ 1,6-hexanediol, 3.5\% triethylene glycol and $40 \mathrm{mM}$ zinc acetate) and $0.6 \mu \mathrm{L}$ of $1.0 \% \mathrm{OG}$ in water. The crystallization trays were then placed in an incubator at $34^{\circ} \mathrm{C}$ to allow bicelles to form gel-like phase, and square shaped thin crystals appeared within a few days. To grow thicker crystals, the coverslips were transferred onto wells with solution containing slightly lowered precipitant concentration (2.4-2.6 $\left.\mathrm{M} \mathrm{NaH}_{2} \mathrm{PO}_{4}\right)$ to enhance the lateral growth of crystals for one more week. Crystals were then harvested and flash frozen in liquid nitrogen after being soaked in the well solution containing $10 \%$ ethylene glycol as a cryoprotectant. For Raman spectroscopy, crystals were independently harvested without freezing and diluted with well solution containing $3.0 \mathrm{M} \mathrm{NaH}_{2} \mathrm{PO}_{4}$.

Data collection and structure determination. X-ray diffraction data were collected at beamline 23ID-D of the Advanced Photon Source (APS) synchrotron at $100 \mathrm{~K}$, with a Pilatus $6 \mathrm{M}$ detector. The crystals were exposed to a $20-\mu \mathrm{m}$-wide, 20 - $\mu \mathrm{m}$-high beam for $0.2 \mathrm{~s}$ at $0.2^{\circ}$ oscillation/frame with no attenuation at a wavelength of $1.0331 \AA$. Diffraction images from three crystals were processed using the software package available at the beamline and merged by Aimless in the CCP4 software package. The structure of XR (PDB entry 3DDL) was used as a search model for molecular replacement with Balbes ${ }^{58}$, and the model was subjected to maximum-likelihood refinement with Refmac5 with manual model building using Coot in the CCP4 software package and further refinement using Phenix ${ }^{59,60}$. The data-collection and refinement statistics are summarized in Table 1.

Size exclusion chromatography. Size exclusion chromatography was used for both preparative and analytical purposes. All runs were performed using an ÄKTA purifier with a Superdex 200 10/300 GL gel filtration column (GE Healthcare). Briefly, for the oligomerization analysis, the column was equilibrated with a buffer containing either $20 \mathrm{mM}$ Tris- $\mathrm{HCl} \mathrm{pH} 8.0,300 \mathrm{mM} \mathrm{NaCl}$ and $0.04 \% \mathrm{DDM}$, or $20 \mathrm{mM}$ sodium citrate $\mathrm{pH} 3.0,300 \mathrm{mM}$ $\mathrm{NaCl}$ and $0.04 \% \mathrm{DDM}$.

Negative-stain EM imaging. A droplet of $3.5 \mu \mathrm{L}$ of purified GR at a concentration of around $0.05 \mathrm{mg} \mathrm{mL}^{-1}$ was applied to glow-discharged carbon coated copper grids (TED PELLA, CAT\# 01840-F) and negatively stained with $2 \%$ uranyl acetate. Images were collected on a Talos L120C transmission electron microscope at the Microscopy Imaging Laboratory at the University of Toronto. 
Raman spectroscopy. Raman spectroscopy of GR crystals was performed using FRA106/s accessory of the Bruker IFS66vs spectrometer, with Nd-YAG laser excitation at $1064 \mathrm{~nm}$, at a $2 \mathrm{~cm}^{-1}$ resolution. Five microliters of crystal suspension in the crystallization buffer was placed in a metallic holder, and 7000 spectra were averaged. Spectrum of the crystallization buffer was recorded under the same conditions and subtracted from the crystals' spectrum.

Time-resolved laser spectroscopy. Time-resolved laser spectroscopy in the visible range (flash-photolysis) was performed using a custom-built single-wavelength spectrometer described previously ${ }^{61}$. The GR crystal suspension was diluted with crystallization buffer to produce an optically transparent sample and the photocycle was initiated with $7 \mathrm{~ns}$ pulses of the second harmonic of an Nd-YAG laser at $532 \mathrm{~nm}$ (Continuum Minilite II, Energy density at the sample $\sim 2 \mathrm{~mJ} / \mathrm{cm}^{2}$ ). Absorption changes of the monochromatic light (provided by an Oriel QTH source and two monochromators) were followed using an Oriel photomultiplier, an amplifier with a $350 \mathrm{MHz}$ bandwidth, and a Gage AD converter (CompuScope 12100-64 M). The kinetic traces were averaged (1000 traces) and converted into a quasi-logarithmic time scale using in-house software ${ }^{62}$.

Site-directed mutagenesis and spin-labeling of GR mutant. Wild-type GR has no reactive cysteine residues for spin-labeling. Hence, a G67C mutation was introduced by PCR using a QuikChange Lightning (Agilent) mutagenesis kit. The site G67 was selected based on the fact that cysteine replacement and spin labeling of the corresponding residue in BPR (S55) had no influence on the multimerization of $\mathrm{BPR}^{45}$. The sequence of the mutant was confirmed by DNA sequencing (ACGT Corporation, Toronto, ON). Purified GR mutant G67C was mixed with $100 \mu \mathrm{M}$ of 1-oxyl-2,2,5,5-tetramethyl- $\Delta 3$-pyrroline-3-methyl methanethiosulfonate (Toronto Research Chemicals) and incubated at room temperature for $30 \mathrm{~min}$ to form spin-labeled GR-67R1. The protein stability and pH-dependent oligomerization of the G67C mutant were confirmed by absorbance spectra and SEC peak profiles throughout the sample preparation process. Excess spin label was removed, and buffer was exchanged to that with desired composition using a $30 \mathrm{kDa}$ MWCO concentrator (Amicon).

CW-EPR measurements. X-band continuous wave (CW)-EPR data of the spin-labeled GR-67R1 at different buffer compositions were acquired using a Bruker ELEXSYS E500 spectrometer equipped with a Bruker EP $041 \mathrm{MR}$ microwave bridge. Samples were loaded into $0.6 \mathrm{~mm}$ ID and $0.84 \mathrm{~mm}$ OD capillaries and inserted into an ER 4123D dielectric resonator for measurement. The field sweep for data collection was 100-G, modulation amplitude was $2-\mathrm{G}$, and the incident microwave power was $0.5029 \mathrm{~mW}$. Data sets were averages of $30 \mathrm{scans}$. Each CW-EPR measurement was acquired at room temperature.

DEER measurements. For DEER measurements, deuterated glycerol was added to the samples as a cryo-protectant (final concentration 20\%). Spin-labeled GR-67R1 was loaded into quartz capillaries (1.5 mm ID and $1.8 \mathrm{~mm} \mathrm{OD}$ ) and flash frozen using a dry ice/ethanol bath. After freezing, the capillaries were loaded into an ER 5107D2 Q-band flexline resonator and Q-band measurements were performed at $80 \mathrm{~K}$ on a Bruker Elexsys 580 spectrometer with a Super Q-FTu Bridge. A 32-ns $\pi$-pump pulse was applied to the low field peak of the nitroxide field swept spectrum, and the observer $\pi / 2(16 \mathrm{~ns})$ and $\pi(32 \mathrm{~ns})$ pulses were positioned $50 \mathrm{MHz}(17.8 \mathrm{G})$ upfield, which corresponds to the nitroxide center line. Distance distributions were obtained from the raw data using the LabVIEW (National Instruments) program "LongDistances" [developed by Christian Altenbach and Wayne Hubbell, University of California, Los Angeles (UCLA)] that can be downloaded from http://www.biochemistry.ucla.edu/biochem/Faculty/Hubbell/. The DEER distance peak simulations were conducted with the open-source package Multiscale Modeling of Macromolecules (MMM) ${ }^{63}$.

Accession number. Coordinates and structure factors for Gloeobacter Rhodopsin have been deposited in the Protein Data Bank with accession number 6NWD.

\section{Data Availability}

The datasets generated during and/or analysed during the current study are available from the corresponding author on reasonable request.

\section{References}

1. Ernst, O. P. et al. Microbial and animal rhodopsins: structures, functions, and molecular mechanisms. Chemical reviews 114, 126-163, https://doi.org/10.1021/cr4003769 (2014).

2. Spudich, J. L., Yang, C. S., Jung, K. H. \& Spudich, E. N. Retinylidene Proteins: Structures and Functions from Archaea to Humans. Annual review of cell and developmental biology 16 (2000).

3. Kurihara, M. \& Sudo, Y. Microbial rhodopsins: wide distribution, rich diversity and great potential. Biophys Physicobiol 12, 121-129, https://doi.org/10.2142/biophysico.12.0_121(2015).

4. Kandori, H. Ion-pumping microbial rhodopsins. Front Mol Biosci 2, 52, https://doi.org/10.3389/fmolb.2015.00052 (2015).

5. Cao, Z. et al. Novel expression and characterization of a light driven proton pump archaerhodopsin 4 in a Halobacterium salinarum strain. Biochimica et biophysica acta 1847, 390-398, https://doi.org/10.1016/j.bbabio.2014.12.008 (2015).

6. Klyszejko, A. L. et al. Folding and assembly of proteorhodopsin. Journal of molecular biology 376, 35-41, https://doi.org/10.1016/j. jmb.2007.11.030 (2008).

7. Sapra, K. T., Besir, H., Oesterhelt, D. \& Muller, D. J. Characterizing molecular interactions in different bacteriorhodopsin assemblies by single-molecule force spectroscopy. Journal of molecular biology 355, 640-650, https://doi.org/10.1016/j.jmb.2005.10.080 (2006).

8. Hussain, S., Kinnebrew, M., Schonenbach, N. S., Aye, E. \& Han, S. Functional consequences of the oligomeric assembly of proteorhodopsin. Journal of molecular biology 427, 1278-1290, https://doi.org/10.1016/j.jmb.2015.01.004 (2015).

9. Ran, T. et al. Cross-protomer interaction with the photoactive site in oligomeric proteorhodopsin complexes. Acta crystallographica. Section D, Biological crystallography 69, 1965-1980, https://doi.org/10.1107/S0907444913017575 (2013).

10. Kovalev, K. et al. Structure and mechanisms of sodium-pumping KR2 rhodopsin. Sci Adv 5, eaav2671, https://doi.org/10.1126/ sciadv.aav2671 (2019) 
11. Brown, L. S. \& Ernst, O. P. Recent advances in biophysical studies of rhodopsins - Oligomerization, folding, and structure. Biochimica et biophysica acta 1865, 1512-1521, https://doi.org/10.1016/j.bbapap.2017.08.007 (2017).

12. Gell, D. A., Grant, R. P. \& Mackay, J. P. The detection and quantitation of protein oligomerization. Advances in experimental medicine and biology 747, 19-41, https://doi.org/10.1007/978-1-4614-3229-6_2 (2012).

13. Essen, L., Siegert, R., Lehmann, W. D. \& Oesterhelt, D. Lipid patches in membrane protein oligomers: crystal structure of the bacteriorhodopsin-lipid complex. Proceedings of the National Academy of Sciences of the United States of America 95, 11673-11678 (1998).

14. Gushchin, I. et al. Crystal structure of a light-driven sodium pump. Nature structural \& molecular biology 22, 390-395, https://doi. org/10.1038/nsmb.3002 (2015).

15. Sasaki, T. et al. Halorhodopsin from natronomonas pharaonis forms a trimer even in the presence of a detergent, dodecyl-beta-Dmaltoside. Photochemistry and photobiology 85, 130-136, https://doi.org/10.1111/j.1751-1097.2008.00406.x (2009).

16. Wang, J., Link, S., Heyes, C. D. \& El-Sayed, M. A. Comparison of the dynamics of the primary events of bacteriorhodopsin in its trimeric and monomeric states. Biophysical journal 83, 1557-1566, https://doi.org/10.1016/S0006-3495(02)73925-8 (2002).

17. Shibata, M. et al. Oligomeric states of microbial rhodopsins determined by high-speed atomic force microscopy and circular dichroic spectroscopy. Scientific reports 8, 8262, https://doi.org/10.1038/s41598-018-26606-y (2018).

18. Shibata, M., Yamashita, H., Uchihashi, T., Kandori, H. \& Ando, T. High-speed atomic force microscopy shows dynamic molecular processes in photoactivated bacteriorhodopsin. Nature nanotechnology 5, 208-212, https://doi.org/10.1038/nnano.2010.7 (2010).

19. Edwards, D. T. et al. Determining the oligomeric structure of proteorhodopsin by Gd3+-based pulsed dipolar spectroscopy of multiple distances. Structure 22, 1677-1686, https://doi.org/10.1016/j.str.2014.09.008 (2014).

20. Milikisiyants, S. et al. Oligomeric Structure of Anabaena Sensory Rhodopsin in a Lipid Bilayer Environment by Combining SolidState NMR and Long-range DEER Constraints. Journal of molecular biology 429, 1903-1920, https://doi.org/10.1016/j. jmb.2017.05.005 (2017).

21. Nakamura, Y. et al. Complete genome structure of Gloeobacter violaceus PCC 7421, a cyanobacterium that lacks thylakoids. DNA Res 10, 137-145 (2003).

22. Choi, A. R., Shi, L., Brown, L. S. \& Jung, K. H. Cyanobacterial light-driven proton pump, gloeobacter rhodopsin: complementarity between rhodopsin-based energy production and photosynthesis. PloS one 9, e110643, https://doi.org/10.1371/journal. pone.0110643 (2014).

23. Jana, S., Eliash, T., Jung, K. H. \& Sheves, M. Retinal Binding to Apo-Gloeobacter Rhodopsin: The Role of $\mathrm{pH}$ and Retinal-Carotenoid Interaction. The journal of physical chemistry. B 121, 10759-10769, https://doi.org/10.1021/acs.jpcb.7b07523 (2017).

24. Vogt, A., Wietek, J. \& Hegemann, P. Gloeobacter rhodopsin, limitation of proton pumping at high electrochemical load. Biophysical journal 105, 2055-2063, https://doi.org/10.1016/j.bpj.2013.08.031 (2013).

25. Tsukamoto, T. et al. Salt bridge in the conserved His-Asp cluster in Gloeobacter rhodopsin contributes to trimer formation. FEBS letters 587, 322-327, https://doi.org/10.1016/j.febslet.2012.12.022 (2013).

26. Mukai, Y., Kamo, N. \& Mitaku, S. Light-induced denaturation of bacteriorhodopsin solubilized by octyl-beta-glucoside. Protein Eng 12, 755-759 (1999).

27. Van Eps, N. et al. Conformational equilibria of light-activated rhodopsin in nanodiscs. Proceedings of the National Academy of Sciences of the United States of America 114, E3268-E3275, https://doi.org/10.1073/pnas.1620405114 (2017).

28. Kato, H. E. et al. Structural basis for $\mathrm{Na}(+)$ transport mechanism by a light-driven $\mathrm{Na}(+)$ pump. Nature 521, 48-53, https://doi. org/10.1038/nature14322 (2015).

29. Tsukamoto, T. et al. X-ray Crystallographic Structure of Thermophilic Rhodopsin: IMPLICATIONS FOR HIGH THERMAL STABILITY AND OPTOGENETIC FUNCTION. The Journal of biological chemistry 291, 12223-12232, https://doi.org/10.1074/jbc. M116.719815 (2016)

30. Hosaka, T. et al. Structural Mechanism for Light-driven Transport by a New Type of Chloride Ion Pump, Nonlabens marinus Rhodopsin-3. The Journal of biological chemistry 291, 17488-17495, https://doi.org/10.1074/jbc.M116.728220 (2016).

31. Shi, L. et al. Three-dimensional solid-state NMR study of a seven-helical integral membrane proton pump-structural insights. Journal of molecular biology 386, 1078-1093 (2009).

32. Reckel, S. et al. Solution NMR structure of proteorhodopsin. Angewandte Chemie 50, 11942-11946, https://doi.org/10.1002/ anie.201105648 (2011).

33. Imasheva, E. S., Balashov, S. P., Choi, A. R., Jung, K. H. \& Lanyi, J. K. Reconstitution of Gloeobacter violaceus rhodopsin with a lightharvesting carotenoid antenna. Biochemistry 48, 10948-10955, https://doi.org/10.1021/bi901552x (2009).

34. Balashov, S. P. et al. Reconstitution of gloeobacter rhodopsin with echinenone: role of the 4-keto group. Biochemistry 49, 9792-9799, https://doi.org/10.1021/bi1014166 (2010).

35. Hashimoto, K., Choi, A. R., Furutani, Y., Jung, K. H. \& Kandori, H. Low-temperature FTIR study of Gloeobacter rhodopsin: presence of strongly hydrogen-bonded water and long-range structural protein perturbation upon retinal photoisomerization. Biochemistry 49, 3343-3350, https://doi.org/10.1021/bi100184k (2010).

36. Ranaghan, M. J., Schwall, C. T., Alder, N. N. \& Birge, R. R. Green proteorhodopsin reconstituted into nanoscale phospholipid bilayers (nanodiscs) as photoactive monomers. Journal of the American Chemical Society 133, 18318-18327, https://doi.org/10.1021/ ja2070957 (2011).

37. Brown, L. S. et al. Glutamic acid 204 is the terminal proton release group at the extracellular surface of bacteriorhodopsin. The Journal of biological chemistry 270, 27122-27126 (1995).

38. Luecke, H., Schobert, B., Richter, H. T., Cartailler, J. P. \& Lanyi, J. K. Structure of bacteriorhodopsin at 1.55 A resolution. Journal of molecular biology 291, 899-911, https://doi.org/10.1006/jmbi.1999.3027 (1999).

39. Miranda, M. R. et al. The photocycle and proton translocation pathway in a cyanobacterial ion-pumping rhodopsin. Biophysical journal 96, 1471-1481, https://doi.org/10.1016/j.bpj.2008.11.026 (2009).

40. Dioumaev, A. K. et al. Proton transfers in the photochemical reaction cycle of proteorhodopsin. Biochemistry 41, 5348-5358 (2002).

41. Imasheva, E. S., Balashov, S. P., Wang, J. M. \& Lanyi, J. K. pH-dependent transitions in xanthorhodopsin. Photochemistry and photobiology 82, 1406-1413, https://doi.org/10.1562/2006-01-15-RA-776 (2006).

42. Miranda, M. R. Biophysical Characterization of a New Group of Rhodopsins from Photosynthetic Organisms. Order No. NR64502 University of Guelph (2010).

43. Dencher, N. A. \& Heyn, M. P. Bacteriorhodopsin monomers pump protons. FEBS letters 108, 307-310 (1979).

44. Kovacs, I., Hollosnagy, K. \& Varo, G. Dark-Adaptation and Spectral Changes in Triton-X-100-Treated Bacteriorhodopsin. J Photoch Photobio B 27, 21-25, https://doi.org/10.1016/1011-1344(94)07050-X (1995).

45. Stone, K. M. et al. Structural insight into proteorhodopsin oligomers. Biophysical journal 104, 472-481, https://doi.org/10.1016/j. bpj.2012.11.3831 (2013).

46. Man, D. et al. Diversification and spectral tuning in marine proteorhodopsins. The EMBO journal 22, 1725-1731, https://doi. org/10.1093/emboj/cdg183 (2003).

47. Dalmas, O., Hyde, H. C., Hulse, R. E. \& Perozo, E. Symmetry-constrained analysis of pulsed double electron-electron resonance (DEER) spectroscopy reveals the dynamic nature of the KcsA activation gate. Journal of the American Chemical Society 134, 16360-16369, https://doi.org/10.1021/ja3069038 (2012).

48. Brouillette, C. G., McMichens, R. B., Stern, L. J. \& Khorana, H. G. Structure and thermal stability of monomeric bacteriorhodopsin in mixed phospholipid/detergent micelles. Proteins 5, 38-46, https://doi.org/10.1002/prot.340050106 (1989). 
49. Maciejko, J. et al. Visualizing Specific Cross-Protomer Interactions in the Homo-Oligomeric Membrane Protein Proteorhodopsin by Dynamic-Nuclear-Polarization-Enhanced Solid-State NMR. Journal of the American Chemical Society 137, 9032-9043, https:// doi.org/10.1021/jacs.5b03606 (2015).

50. Yoshimura, K. \& Kouyama, T. Structural role of bacterioruberin in the trimeric structure of archaerhodopsin-2. Journal of molecular biology 375, 1267-1281, https://doi.org/10.1016/j.jmb.2007.11.039 (2008).

51. Pebay-Peyroula, E., Rummel, G., Rosenbusch, J. P. \& Landau, E. M. X-ray structure of bacteriorhodopsin at 2.5 angstroms from microcrystals grown in lipidic cubic phases. Science 277, 1676-1681 (1997).

52. Kolbe, M., Besir, H., Essen, L. O. \& Oesterhelt, D. Structure of the light-driven chloride pump halorhodopsin at 1.8 A resolution. Science 288, 1390-1396 (2000).

53. Wang, S. et al. Solid-state NMR spectroscopy structure determination of a lipid-embedded heptahelical membrane protein. Nature methods 10, 1007-1012, https://doi.org/10.1038/nmeth.2635 (2013).

54. Shevchenko, V. et al. Inward H(+) pump xenorhodopsin: Mechanism and alternative optogenetic approach. Sci Adv 3, e1603187, https://doi.org/10.1126/sciadv.1603187 (2017).

55. Kawanabe, A., Furutani, Y., Jung, K. H. \& Kandori, H. Engineering an inward proton transport from a bacterial sensor rhodopsin. Journal of the American Chemical Society 131, 16439-16444, https://doi.org/10.1021/ja904855g (2009).

56. Faham, S. \& Bowie, J. U. Bicelle crystallization: a new method for crystallizing membrane proteins yields a monomeric bacteriorhodopsin structure. Journal of molecular biology 316, 1-6, https://doi.org/10.1006/jmbi.2001.5295 (2002).

57. Faham, S. et al. Crystallization of bacteriorhodopsin from bicelle formulations at room temperature. Protein science: a publication of the Protein Society 14, 836-840, https://doi.org/10.1110/ps.041167605 (2005).

58. Long, F., Vagin, A. A., Young, P. \& Murshudov, G. N. BALBES: a molecular-replacement pipeline. Acta crystallographica. Section D, Biological crystallography 64, 125-132, https://doi.org/10.1107/S0907444907050172 (2008).

59. Emsley, P., Lohkamp, B., Scott, W. G. \& Cowtan, K. Features and development of Coot. Acta crystallographica. Section D, Biological crystallography 66, 486-501, https://doi.org/10.1107/S0907444910007493 (2010).

60. Adams, P. D. et al. PHENIX: a comprehensive Python-based system for macromolecular structure solution. Acta crystallographica. Section D, Biological crystallography 66, 213-221, https://doi.org/10.1107/S0907444909052925 (2010).

61. Sumii, M., Furutani, Y., Waschuk, S. A., Brown, L. S. \& Kandori, H. Strongly hydrogen-bonded water molecule present near the retinal chromophore of Leptosphaeria rhodopsin, the bacteriorhodopsin-like proton pump from a eukaryote. Biochemistry 44, 15159-15166, https://doi.org/10.1021/bi0513498 (2005).

62. Shi, L., Yoon, S. R., Bezerra, A. G. Jr., Jung, K. H. \& Brown, L. S. Cytoplasmic shuttling of protons in anabaena sensory rhodopsin: implications for signaling mechanism. Journal of molecular biology 358, 686-700, https://doi.org/10.1016/j.jmb.2006.02.036 (2006).

63. Polyhach, Y., Bordignon, E. \& Jeschke, G. Rotamer libraries of spin labelled cysteines for protein studies. Physical chemistry chemical physics: PCCP 13, 2356-2366, https://doi.org/10.1039/c0cp01865a (2011).

64. Bordignon, E. Site-directed spin labeling of membrane proteins. Topics in current chemistry 321, 121-157, https://doi. org/10.1007/128_2011_243(2012).

65. Zuckerkandl, E. \& Pauling, L. B. Evolutionary divergence and convergence in proteins. In Evolving Genes and Proteins (eds. Bryson, V. \& Vogel, H. J.) 97-166 (Academic Press, New York, 1965).

66. Kumar, S., Stecher, G. \& Tamura, K. MEGA7: Molecular Evolutionary Genetics Analysis Version 7.0 for Bigger Datasets. Mol Biol Evol 33, 1870-1874, https://doi.org/10.1093/molbev/msw054 (2016).

\section{Acknowledgements}

The authors thank Emil F. Pai and Bryan T. Eger (University of Toronto) for discussions and helpful suggestions about the determination of the GR crystal structure. We also thank Kengo Sasaki (Nagoya Institute of Technology) for the preparation of purified GR protein for the initial crystallization screening. This research used resources of the Advanced Photon Source, a U.S. Department of Energy (DOE) Office of Science User Facility operated for the DOE Office of Science by Argonne National Laboratory under contract no. DE-AC02-06CH11357. We specifically thank the staff at the GM/CA beamlines 23-ID. This work has been supported in part by the Canada Excellence Research Chairs (CERC) program and the Natural Sciences and Engineering Research Council of Canada (NSERC, grant RGPIN-2017-06862). O.P.E. is a CIFAR fellow and holds the Anne and Max Tanenbaum Chair in Neuroscience at the University of Toronto.

\section{Author Contributions}

T.M., W.O. and O.P.E. designed the experiments and wrote the manuscript; T.M. and W.O. performed protein crystallography and EPR measurement with support from N.V.E.; K.I. and H.K. were involved in experimental design and analysis of data; L.S.B. performed Raman and laser spectroscopy. All authors reviewed and edited the manuscript.

\section{Additional Information}

Supplementary information accompanies this paper at https://doi.org/10.1038/s41598-019-47445-5.

Competing Interests: The authors declare no competing interests.

Publisher's note: Springer Nature remains neutral with regard to jurisdictional claims in published maps and institutional affiliations.

(c) (1) Open Access This article is licensed under a Creative Commons Attribution 4.0 International License, which permits use, sharing, adaptation, distribution and reproduction in any medium or format, as long as you give appropriate credit to the original author(s) and the source, provide a link to the Creative Commons license, and indicate if changes were made. The images or other third party material in this article are included in the article's Creative Commons license, unless indicated otherwise in a credit line to the material. If material is not included in the article's Creative Commons license and your intended use is not permitted by statutory regulation or exceeds the permitted use, you will need to obtain permission directly from the copyright holder. To view a copy of this license, visit http://creativecommons.org/licenses/by/4.0/.

(c) The Author(s) 2019 